07

\title{
Квантовое сжатие поля одноатомного лазера в условиях переменной константы связи
}

\author{
(C) В.А. Бобрикова, Р.А. Хачатрян, К.А. Баранцев, Е.Н. Попов \\ Санкт-Петербургский политехнический университет Петра Великого, \\ 195251 Санкт-Петербург, Россия \\ ฯ e-mail: enp-tion@yandex.ru \\ Поступила в редакцию 04.02.2019 г. \\ В окончательной редакции 05.08.2019 г. \\ Принята к публикации 12.08.2019 г.
}

\begin{abstract}
Исследован нестационарный режим генерации сжатого света одноатомным лазером. Численно получены зависимости параметра квантового сжатия и интенсивности излучения от частоты модуляции константы связи атома с полем. Показано, что при частоте модуляции, равной удвоенной средней константе связи, возникает резонанс, который приводит к более эффективному квантовому сжатию в нестационарном гармоническом режиме, чем в случае стационарного режима при тех же самых параметрах релаксации и накачки.
\end{abstract}

Ключевые слова: одноатомный лазер, константа связи, сжатый свет.

DOI: $10.21883 /$ OS.2019.12.48695.39-19

\section{Введение}

Источники света с неклассической статистикой являются одним из трендов развития квантовой оптики и имеют широкие перспективы применения. Так, в метрологии это стандартизация частоты [1,2] и магнитометрия [3,4], в квантово-информационных приложениях это запись и хранение квантовой информации и криптография [5-7]. Задача о генерации света с неклассической статистикой может быть решена разными способами. Так, к примеру, это возможно осуществить в лазерах с помощью определенных статистических свойств накачки [8-14] или/и использованием многоуровневых схем накачки $[15,16]$.

Изолированные одиночные излучатели в силу возможности наблюдения на них эффекта антигруппировки фотонов также являются перспективными системами для получения неклассических состояний света. Так, на практике уже были реализованы лазеры на одиночных атомах [17], ионах [18] и квантовых точках [19]. В этих экспериментах наблюдались субпуассонова статистика излучения, а также подавление амплитудных флуктуаций ниже квантового предела. В теоретических работax [20,21], посвященных изучению фундаментальной модели одноатомного лазера, показано, что при определенном соотношении параметров системы такой лазер может излучать свет, сжатый по амплитуде. Примечательно, что в переходном процессе может наблюдаться переменное во времени сжатие по амплитуде. Это наводит на мысль о возможности увеличения эффективности квантового сжатия поля одноатомного лазера посредством модуляции константы связи атом-поле.

Временную зависимость константы связи можно реализовать разными способами. Один из них - это вынужденные колебания зеркал резонатора. Качественная оценка показывает, что для наблюдения рассматриваемого в работе эффекта частота колебаний зеркал меньше $10^{8} \mathrm{~Hz}$ является достаточной. Такая частота может быть достигнута, например, с помощью пьезоэлектрических манипуляторов. Другой способ состоит в возбуждении вынужденных колебаний одиночного излучателя в оптической решетке, потенциальная яма которой регулируется параметрами удерживающих лучей. Точечный излучатель, который можно с хорошим приближением описывать как двухуровневый атом, в кристаллической решетке может совершать тепловые колебания на определенной частоте, что обусловливает появление дополнительного шума. Другой причиной шума могут стать температурные флуктуации положения зеркал резонатора, которые подробно исследовались в работе [22]. Однако в нашей задаче будем предполагать, что такими флуктуациями можно пренебречь, и временная зависимость константы связи от времени является детерминированной.

\section{Модель одноатомного лазера с модулированной константой связи}

Простейшая фундаментальная модель одноатомного лазера представляет собой двухуровневый атом, который взаимодействует с одиночной выделенной модой резонатора с потерями [23-28]. Динамику системы определяют всего четыре процесса, от которых зависят свойства генерируемого излучения: некогерентная накачка, под действием которой атом переходит из основного состояния $|a\rangle$ в возбужденное $|b\rangle$ со скоростью $Г$; спонтанный распад атома из возбужденного состояния $|b\rangle$ в основное $|a\rangle$, который происходит со скоростью $\gamma$; 
уход фотонов из резонатора, который характеризуется добротностью или обратным временем жизни фотонов в резонаторе $\kappa$; взаимодействие атома с полевой модой, которое характеризуется константой связи $g$.

Уравнение для атомно-полевого оператора плотности одноатомного лазера с некогерентной накачкой имеет следующий вид:

$$
\frac{\partial \hat{\rho}}{\partial t}=-\frac{i}{\hbar}[\hat{V}, \hat{\rho}]+\frac{\kappa}{2} \hat{\mathbf{K}}\{\hat{\rho}\}+\frac{\gamma}{2} \hat{\mathbf{R}}\{\hat{\rho}\}+\frac{\Gamma}{2} \hat{\mathbf{P}}\{\hat{\rho}\}
$$

где первое слагаемое в правой части описывает взаимодействие атома с полевой модой, второе - релаксацию поля, третье - спонтанный распад атома, четвертое некогерентную накачку атома. Ниже приведен явный вид оператора взаимодействия $\hat{V}$ и супероператоров, описывающих соответствующие релаксационные процессы:

$$
\begin{gathered}
\hat{V}=i \hbar g\left(\hat{a}^{\dagger} \hat{\sigma}-\hat{\sigma}^{\dagger} \hat{a}\right), \\
\hat{\mathbf{K}}\{\hat{\rho}\}=2 \hat{a} \hat{\rho} \hat{a}^{\dagger}-\hat{a}^{\dagger} \hat{a} \hat{\rho}-\hat{\rho} \hat{a}^{\dagger} \hat{a}, \\
\hat{\mathbf{R}}\{\hat{\rho}\}=2 \hat{\sigma} \hat{\rho} \hat{\sigma}^{\dagger}-\hat{\sigma}^{\dagger} \hat{\sigma} \hat{\rho}-\hat{\rho} \hat{\sigma}^{\dagger} \hat{\sigma}, \\
\hat{\mathbf{P}}\{\hat{\rho}\}=2 \hat{\sigma}^{\dagger} \hat{\rho} \hat{\sigma}-\hat{\sigma} \hat{\sigma}^{\dagger} \hat{\rho}-\hat{\rho} \hat{\sigma} \hat{\sigma}^{\dagger} .
\end{gathered}
$$

Здесь $\hat{a}, \hat{a}^{\dagger}$ - операторы уничтожения и рождения фотона в моде резонатора, $\hat{\sigma}, \hat{\sigma}^{\dagger}-$ операторы дипольного атомного перехода. Для исследования решения уравнения (1) перепишем полевые операторы в базисе фоковских состояний, а атомные - в базисе стационарных состояний невозмущенного атома:

$$
\begin{gathered}
\hat{a}=\sum_{n=1}^{\infty} \sqrt{n}|n-1\rangle\langle n|, \hat{\sigma}=| a\rangle\langle b|, \\
\hat{a}^{\dagger}=\sum_{n=0}^{\infty} \sqrt{n+1}|n+1\rangle\left\langle n\left|, \quad \hat{\sigma}^{\dagger}=\right| b\right\rangle\langle a| .
\end{gathered}
$$

Подобная форма записи операторов рождения и уничтожения удобна при проведении вычислений, так как задает явный вид матричных элементов операторов. Тогда уравнение для матрицы плотности одноатомного лазера $\rho_{\alpha \beta}^{n m}=\langle n, \alpha|\hat{\rho}| m, \beta\rangle$, где $|n, \alpha\rangle=|n\rangle|\alpha\rangle, \alpha, \beta=a, b$, $n=0,1,2, \ldots, \infty$, можно представить как бесконечную систему дифференциальных уравнений:

$$
\begin{aligned}
& \frac{\partial \rho_{a a}^{n m}}{\partial t}=g\left(\sqrt{n} \rho_{b a}^{n-1, m}+\sqrt{m} \rho_{a b}^{n, m-1}\right)-\frac{\kappa}{2}(n+m) \rho_{a a}^{n m} \\
& +\gamma \rho_{b b}^{n m}-\Gamma \rho_{a a}^{n m}+\kappa \sqrt{(n+1)(m+1)} \rho_{a a}^{n+1, m+1} \\
& \frac{\partial \rho_{b b}^{n m}}{\partial t}=-g\left(\sqrt{m+1} \rho_{b a}^{n, m+1}+\sqrt{n+1} \rho_{a b}^{n+1, m}\right)-\frac{\kappa}{2}(n+m) \\
& \times \rho_{b b}^{n m}+\kappa \sqrt{(n+1)(m+1)} \rho_{b b}^{n+1, m+1}-\gamma \rho_{b b}^{n m}+\Gamma \rho_{a a}^{n m},
\end{aligned}
$$

$$
\begin{aligned}
& \frac{\partial \rho_{a b}^{n m}}{\partial t}=g\left(\sqrt{n} \rho_{b b}^{n-1, m}-\sqrt{m+1} \rho_{a a}^{n, m+1}\right)-\frac{\kappa}{2}(n+m) \\
& \times \rho_{a b}^{n m}-\frac{\gamma+\Gamma}{2} \rho_{a b}^{n m}+\kappa \sqrt{(n+1)(m+1)} \rho_{a b}^{n+1, m+1}, \\
& \frac{\partial \rho_{b a}^{n m}}{\partial t}=g\left(\sqrt{m} \rho_{b b}^{n, m-1}-\sqrt{n+1} \rho_{a a}^{n+1, m}\right)-\frac{\kappa}{2}(n+m) \\
& \times \rho_{b a}^{n m}-\frac{\gamma+\Gamma}{2} \rho_{b a}^{n m}+\kappa \sqrt{(n+1)(m+1)} \rho_{b a}^{n+1, m+1} .
\end{aligned}
$$

Как и в работе [21] для решения бесконечной системы дифференциальных уравнений (8)-(11) базис состояний поля ограничивался, после чего проводилось численное решение системы. Установившийся режим генерации одноатомного лазера наиболее интересен с практической точки зрения, который в случае постоянных параметров квантовой системы $(\Gamma, \gamma, \kappa, g)$ является стационарным режимом. Для характеристики квантовой статистики поля после завершения переходных процессов можно определить среднее число фотонов $\langle n\rangle$ в резонаторе и степень сжатия света в стационарном режиме, которая описывается параметром Манделя $Q$ :

$$
\begin{gathered}
\langle n\rangle=\sum_{n=0}^{k} n\left(\rho_{a a}^{n n}+\rho_{b b}^{n n}\right)=\sum_{n=0}^{k} n \rho^{n n}, \\
Q=\frac{\left\langle n^{2}\right\rangle-\langle n\rangle^{2}}{\langle n\rangle}-1,
\end{gathered}
$$

где $k$ - значение числа заполнения, на котором искусственно обрывается система уравнений, $\rho^{n n}-$ диагональный элемент матрицы плотности, усредненной по двум состояниям атома, он характеризует вероятность нахождения $n$ фотонов в полевой моде резонатора. В случае, когда параметр $Q$ становится отрицательным, свет приобретает неклассические свойства и имеет субпуассонову квантовую статистику.

Введем в уравнениях (8)-(11) временную зависимость константы связи, которая соответствует гармонической функции:

$$
g=g_{0}+g_{1} \sin \left(\Omega t+\varphi_{1}\right),
$$

где $g_{1}$ - амплитуда модуляции, $\Omega$ - частота модуляции константы связи, $\varphi_{1}-$ некоторая постоянная фаза, $g_{0}$ - не зависящая от времени часть. Причем для функции (14) принято условие $g_{1} / g_{0} \ll 1$.

Выбор малой амплитуды модуляции по сравнению с постоянной составляющей константы связи продиктован тем, что нас интересует в первую очередь отличие стационарного режима генерации одноатомного лазера от нестационарного режима, который проще рассматривать в качестве линейного возмущения. В противном случае при большой амплитуде модуляции $g_{1}$ могут наблюдаться сильные переходные процессы от стационарного режима при максимальном значении константы связи 
$g=g_{0}+g_{1}$ к стационарному решению при минимальном значении $g=g_{0}-g_{1}$. Они не несут в себе интересной информации, но существенно усложняют динамику поля в резонаторе. Гармоническая функция константы связи (14) обусловлена поиском резонансных эффектов, которые могут возникать в нестационарном режиме генерации одноатомного лазера. Точно определенная начальная фаза $\varphi_{1}$, стоящая в аргументе синуса (14), запускает механизм фазовой синхронизации и позволяет преодолеть разрушение сжатого состояния вследствие диффузии фазы.

\section{Результаты}

Предварительный анализ динамки среднего числа фотонов $\langle n\rangle$ и параметра $Q$ при постоянных константах $\Gamma, \gamma, \kappa, g$ позволяет утверждать, что обратное время переходного процесса можно оценить как сумму скоростей $\Gamma, \gamma, \kappa$ некогерентных процессов в модели, а период осцилляций диагональных элементов атомнополевой матрицы плотности во время переходного процесса зависит от постоянной составляющей константы связи $g_{0}$. Для наблюдения резонансного эффекта период модуляции $g$ должен быть меньше или сопоставим с временем переходного процесса. В противном случае динамика будет соответствовать адиабатическому режиму, при котором установление равновесия происходит быстрее изменения параметров системы. Поэтому в качестве исходных параметров при поиске резонансного эффекта были выбраны параметры одноатомного лазера из работы [20]:

$$
\Gamma=1.4 g_{0}, \kappa=1.4 g_{0}, \quad \gamma=0.1 g_{0} .
$$

Эти параметры удовлетворяют обозначенным требованиям и приводят к наибольшей степени сжатия в стационарном режиме, что делает их наиболее перспективными с точки зрения ожидаемого результата расчетов.

На рис. 1 и 2 приведены зависимости среднего числа фотонов в резонаторе и параметра Манделя $Q$ от частоты модуляции $\Omega$ при параметрах одноатомного лазера (15). Поскольку при временной зависимости константы связи (14) система дифференциальных уравнений (8)-(11) не имеет стационарного решения, то результаты представлены парами кривых: максимальное и минимальное за период модуляции значение исследуемой величины в установившейся динамике. Индексы min и max на графиках обозначают наименьшее и наибольшее значения параметра $Q$ или среднего числа фотонов, которые периодически достигаются в нестационарном режиме генерации одноатомного лазера.

Зависимости параметра $Q$ от частоты модуляции содержат характерный экстремум (рис. 2), который свидетельствует о наличии параметрического резонанса в модели одноатомного лазера. Максимум резонансной кривой достигается при частоте модуляции $\Omega \approx 2 g$, и

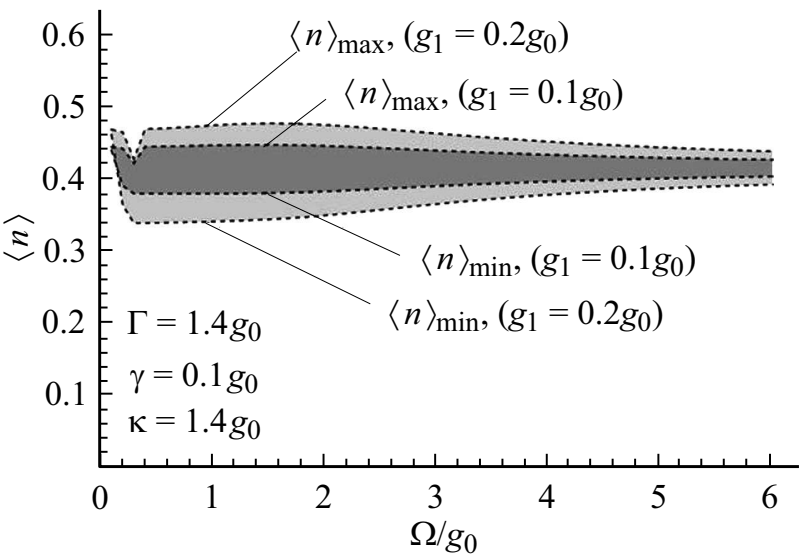

Рис. 1. Зависимость среднего числа фотонов от частоты модуляции константы связи при двух различных значениях параметра $g_{1}$ из формулы (14).

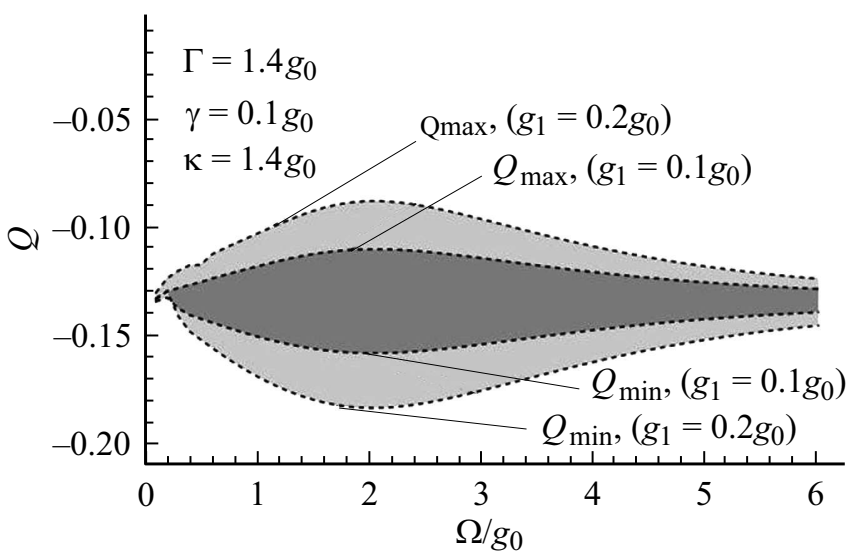

Рис. 2. Зависимости параметра Манделя $Q$ от частоты модуляции константы связи при двух различных значениях параметра $g_{1}$ из формулы (14).

его положение по оси абсцисс практически не отличается в случае различных констант $\gamma, \kappa$ и Г, что было проверено с помощью численного решения системы (8)(11). Максимум на частотной кривой, соответствующей среднему числу фотонов $\langle n\rangle$, не выражен так же ярко, как для параметра $Q$ (рис. 1$)$.

Для наблюдения установившейся динамики состояния поля нами были построены временные зависимости среднего числа фотонов, параметра $Q$ и вероятностей нескольких „фоковских“ состояний для нестационарного режима генерации (рис. 3-5), когда параметр $g$ определяется формулой (14). Отметим, что на этих рисунках нанесены дополнительные динамические кривые, соответствующие стационарному режиму генерации с наибольшим $g_{\text {const }}=g_{0}+g_{1}$ и наименьшим $g_{\text {const }}=g_{0}-g_{1}$ значениями константы связи $g$, которые достигаются за период модуляции.

Неожиданным результатом является тот факт, что отличие параметра $Q$ для стационарного режима с наибольшим и наименьшим значениями постоянной кон- 


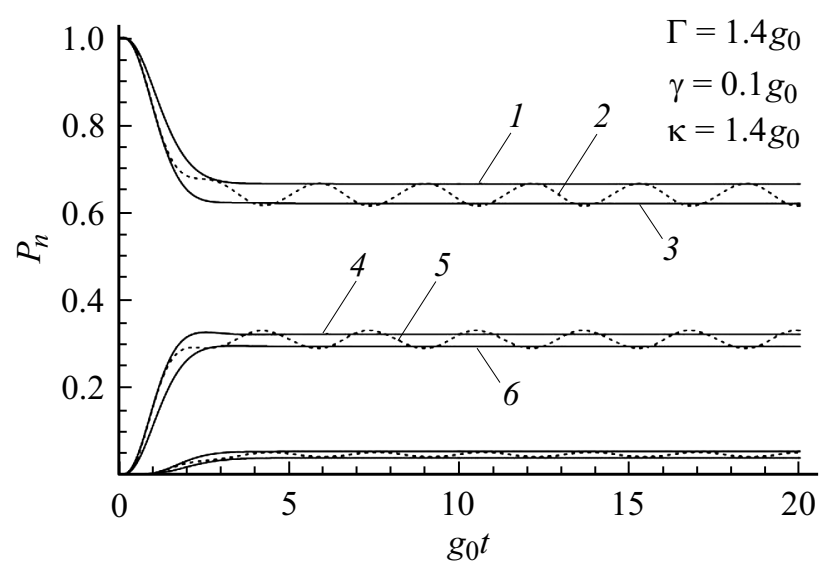

Рис. 3. Зависимость вероятности $P_{n}$ нахождения $n$ фотонов в резонаторе одноатомного лазера от времени: (1) $P_{0}$ в стационарном режиме при $g_{\text {const }}=0.9 g_{0}$, (2) $P_{0}$ в нестационарном режиме (14) при $g=g_{0}\left(1+0.1 \sin \left(2 g_{0} t\right)\right)$, (3) $P_{0}$ в стационарном режиме при $g_{\text {const }}=1.1 g_{0}$, (4) $P_{1}$ в стационарном режиме при $g_{\text {const }}=0.9 g_{0},(5) P_{1}$ в нестационарном режиме (14) при $g=g_{0}\left(1+0.1 \sin \left(2 g_{0} t\right)\right)$, (6) $P_{1}$ в стационарном режиме при $g_{\text {const }}=1.1 g_{0}$, три нижние кривые построены по аналогии с верхними.

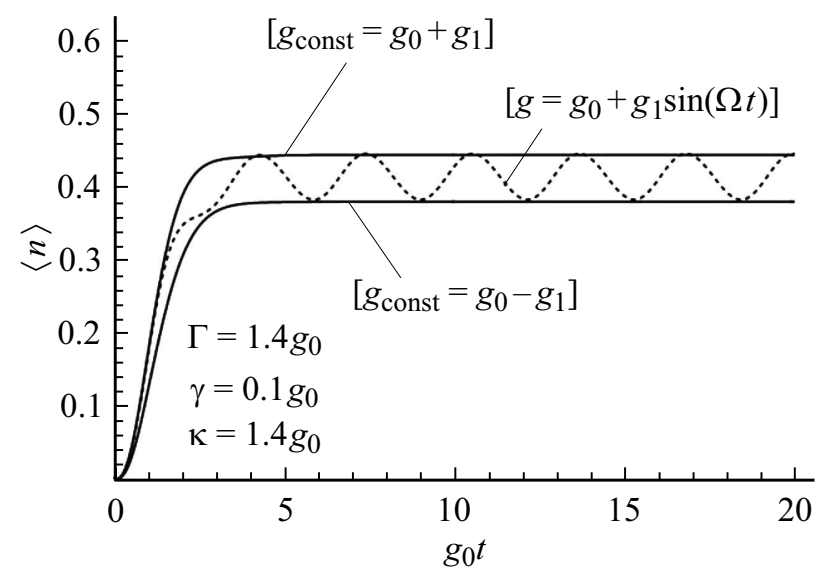

Рис. 4. Зависимость среднего числа фотонов от времени при разных константах связи, соответствующих стационарному и нестационарному режимам генерации. Константы $g_{1}$ и $\Omega$ определены в формуле (14): $g_{1}=0.1 g_{0}, \Omega=2 g_{0}$.

станты связи $g$ на порядок меньше изменения параметра $Q$ за период модуляции константы связи в этом же диапазоне, но в нестационарном режиме генерации (рис. 5). Ниже приведены численные значения параметра Манделя $Q$, соответствующие рис. 5:

$$
\begin{gathered}
Q_{g=0.9}^{(s)}=-0.132, Q_{g=1.1}^{(s)}=-0.134, \\
Q_{\min }=-0.159, Q_{\max }=-0.111, \\
\frac{\left|Q_{\min }-Q_{\max }\right|}{\left|Q_{g=0.9}^{(s)}-Q_{g=1.1}^{(s)}\right|}=2.25,
\end{gathered}
$$

где $Q_{g=0.9}^{(s)}$ и $Q_{g=1.1}^{(s)}$ - параметры Q поля одноатомного лазера в стационарном режиме при постоянных кон- стантах связи $g=0.9$ и $g=1.1$ соответственно, $Q_{\min }$ и $Q_{\max }$ - это минимальное и максимальное значения параметра $Q$ в нестационарном режиме за период модуляции константы связи (14) в диапазоне между $g=0.9$ и $g=1.1$ соответственно.

Следует отметить, что временная зависимость среднего числа фотонов $\langle n\rangle$ не обладает подобным свойством, т.е. разность между наибольшим и наименьшим значениями для двух стационарных режимов примерно равна амплитуде модуляции в случае нестационарного режима генерации (рис. 4).

Из (16)-(18) следует, что нестационарный режим генерации не может быть рассмотрен как адиабатическая зависимость стационарного режима от меняющихся параметров системы. В противном случае разность между максимальным и минимальным значением параметра $Q$ отличалась бы незначительно при разных частотах модуляции константы связи $\Omega$. Это говорит о существовании сильного нелинейного резонансного эффекта. Мы предполагаем, что его можно использовать для достижения большего, чем в стационарном режиме, сжатия света, генерируемого в резонаторе одноатомного лазера. Отметим, что для использования генерации сжатого света в установившемся динамическом режиме необходимо использование синхронного детектора на фотоэлементе, иначе накопленная за длительный по сравнению с периодом модуляции промежуток времени статистика будет усреднена по сильным, но частым колебаниям среднего числа фотонов в резонаторе.

\section{Заключение}

Предсказано существование нелинейного резонансного эффекта генерации сжатого света в резонаторе одноатомного лазера с модулированной константой связи. Найдена зависимость параметра Манделя $Q$ и среднего числа фотонов в резонаторе от времени при входных

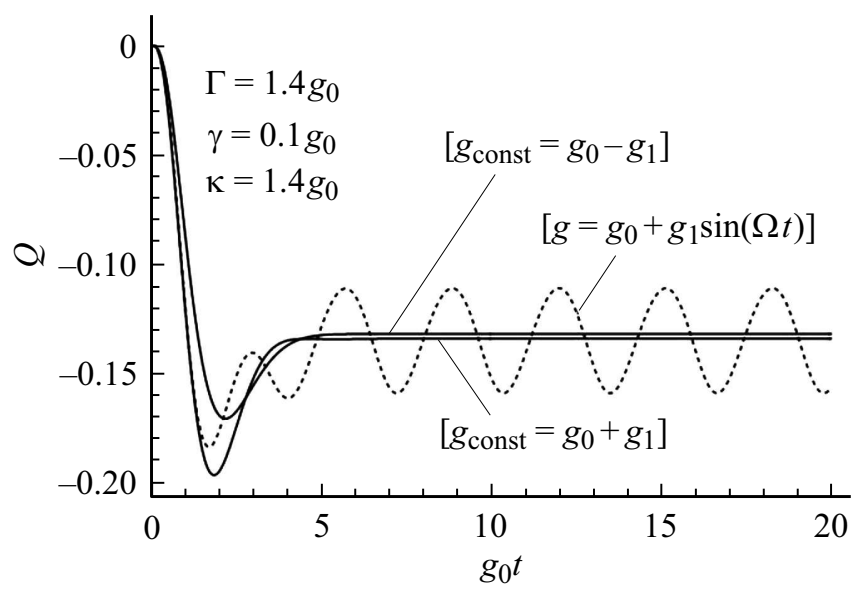

Рис. 5. Зависимость параметра Манделя $Q$ от времени при разных константах связи, соответствующих стационарному и нестационарному режимам генерации. Константы $g_{1}$ и $\Omega$ определены в формуле (14): $g_{1}=0.1 g_{0}, \Omega=2 g_{0}$. 
параметрах одноатомного лазера, приводящих к наибольшему сжатию при стационарном режиме генерации. На основании картины установившейся динамики были построены частотные кривые зависимости максимального и минимального значений параметра Манделя $Q$ и среднего числа фотонов $\langle n\rangle$ от частоты модуляции константы связи $g$ в случае низкодобротного резонатора. Численно предсказан эффект увеличения эффективности сжатия света в нестационарном режиме генерации по сравнению со стационарным режимом работы одноатомного лазера.

\section{Финансирование работы}

Работа выполнена при финансовой поддержке РФФИ (грант № 18-32-00250).

\section{Конфликт интересов}

Авторы заявляют, что у них нет конфликта интересов.

\section{Список литературы}

[1] Merimaa M., Lindvall T., Tittonen I., Ikonen E. // J. Opt. Soc. Am. B. 2003. V. 20. N 2. P. 273-279.

[2] Vanier J. // Appl. Phys. B. 2005. V. 81. N 4. P. 421-442.

[3] Stahler M., Wynands R., Knappe S. et al. // Opt. Lett. 2002. V. 27. N 16. P. $1472-1474$.

[4] Akulshin A., Celikov A., Velichansky V. // Opt. Commun. 1991. V. 84. N 3-4. P. 139-143.

[5] Lucin M.D. // Rev. Mod. Phys. 2003. V. 75. N 2. P. 457-472.

[6] Fleischhauer M., Imamoglu A., Marangos J.P. // Rev. Mod. Phys. 2005. V. 77. N 2. P. 633-673.

[7] Килин С.Я. // УФН. 1999. Т. 169. № 5. С. 507-527.

[8] Голубев Ю.М., Соколов И.В. // ЖЭТФ. 1984. Т. 87. № 8. C. 408.

[9] Yamamoto Y., Mashida S., Nilson O. // Phys. Rev. A. 1986. V. 34. P. 4025.

[10] Benkert C., Scully M.O., Bergou J. etal. // Phys. Rev. A. 1990. V. 41. P. 2756.

[11] Reshetov V.A., Popov E.N., Yevseyev I.V. // Las. Phys. Lett. 2010. V. 7. P. $218-224$.

[12] Kolobov M.I., Davidovich L., Giacobino E., Fabre C. // Phys. Rev. A. 1993. V. 47. N 2. P. 1431-1446.

[13] Козловский А.В. // ЖЭТФ. 1993. Т. 104. С. 2995-3007.

[14] Eschmann A., Gardiner C.W. // Phys. Rev. A. 1996. V. 54. P. 3373-3380.

[15] Marte M.A., Ritsch H., Walls D.F. // Phys. Rev. Lett. 1988. V. 61. N 9. P. 1093-1096.

[16] Kennedy T.A.B., Walls D.F. // Phys Rev. A. 1989. V. 40. N 11. P. 6366-6373.

[17] McKeever J., Boca A., Boozer A.D. etal. // Nature (London). 2003. V. 425. P. 268-271.

[18] Dubin F., Russo C., Barros H.G., Stute A., Becher C., Schmidt P.O., Blatt R. // Nat. Phys. 2010. V. 6. P. 350-353.

[19] Nomura M., Kumagai N., Iwamoto S., Ota Y., Arakawa Y. // Opt. Express. 2009. V. 17. N 18. P. 15975-15982.

[20] Козловский А.В., Ораевский А.Н. // ЖЭТФ. 1999. Т. 115. № 4. C. 1210-1220.
[21] Ларионов Н.В., Баранцев К.А., Попов Е.Н. // Научнотехнические ведомости СПбПУ. Физико-математические науки. 2018. Т. 11. № 4. С. 104-111.

[22] Heinert D., Craig K., Grote H. etal. // Rhys. Rev. D. 2014. V. 90. P. 042001

[23] Larionov N.V., Kolobov M.I. // Phys. Rev. A. 2013. V. 88. N 1. P. 013843.

[24] Popov E.N., Larionov N.V. // Proc. SPIE. 2016. V. 9917. P. $1-$ 6.

[25] Larionov N.V., Kolobov M.I. // Phys. Rev. A. 2011. V. 84. N 5. P. 055801.

[26] Карлович Т.Б., Килин С.Я. // Опт. и спектр. 2001. Т. 91. C. 375-383; Karlovich T.B., Kilin S.Ya. // Opt. Spectrosc. 2001. V. 91 . N 3. P. $343-351$.

[27] Карлович Т.Б., Килин С.Я. // Опт. и спектр. 2007. Т. 103. C. 260-271; Karlovich T.B., Kilin S.Ya. // Opt. Spectrosc. 2007. V. 103. N 2. P. $280-290$.

[28] Kilin S.Ya., Mikhalychev A.B. // Phys. Rev. A. 2012. V. 85. N 6. P. 063817. 